\title{
Through the wall radar imaging with MIMO beamforming processing - simulation and experimental results
}

\author{
B. Boudamouz ${ }^{1}$, P. Millot ${ }^{1}$, C. Pichot ${ }^{2}$ \\ ${ }^{1}$ ONERA, The French Aerospace Lab, DEMR (Département d'Electromagnétisme et Radar), F31055 Toulouse, France \\ ${ }^{2}$ Laboratoire Electronique Antennes Télécommunications, LEAT-CNRS Université de Nice-Sophia Antipolis. Nice, France
}

\section{Email address:}

brahim.boudamouz@onera.fr (B. Boudamouz), patrick.millot@onera.fr (P. Millot), christian.pichot@unice.fr (C. Pichot)

To cite this article:

B. Boudamouz, P. Millot, C. Pichot. Through the Wall Radar Imaging with MIMO beamforming processing - Simulation and Experimental Results, American Journal of Remote Sensing. Vol. 1, No. 1, 2013, pp. 7-12. doi: 10.11648/j.ajrs.20130101.12

\begin{abstract}
In this paper, we address the problem of Through The Wall (TTW) detection with the emerging radar concept that is the Multiple-Input Multiple-Output (MIMO) radar. At first, near field and through the wall propagation effects on electromagnetic waves are adressed then a MIMO frequency signal model is given and the advantages of a MIMO scheme for the complex task of TTW detection is discussed. Then image formation with beamforming processing is derived. Finally, the proposed imaging method is implemented on numerical signals obtained by FDTD computations considering through cinder blocks walls propagation. The obtained images for a scenario with one or two targets are presented. Then the experimental setup is exposed and the realised MIMO radar prototype is described. Finally, experimental results of radar imaging through a cinder block wall are shown.
\end{abstract}

Keywords: Through The Wall Radar Imaging; Beamforming Processing, MIMO Radar Experimentation

\section{Introduction}

Urban sensing and more particularly TTW imaging has become an important field of research and developments [2], [3] due to its great potentials in terms of civilian and military applications. In fact TTW systems could allow law enforcement officials and military agents to adapt strategies in hostage taking scenarii and avoid risks on the field. Evolved sytems could also be used to detect human being in fire buildings and quake rubble and then give help for rescue operations.

Among the various existing technologies allowing TTW detection, it seems that the most adequate and mature ones are those based on radar principles. First works date from the last past decades [4] and processing techniques were derived from Ground Penetrating Radar (GPR) but afterwards general radar processing techniques have been implemented for TTW sensing. Detection and localization in TTW environment is a really tough task because its effects on electromagnetic propagation as phase distorsion and delay. Some of the realized works in TTW detection can be found in [5], [6] and references given inside. Several works try to take into account those effects and use retro-propagation processing or time-reversal methods [7] to image the scanned zone. Some others used super resolution processing to improve the resolution of the image formed [8] but still few use MIMO radar processing for TTW imaging.

Here we present the advantages of a MIMO architecture for the general task of TTW detection. Then we derive MIMO conventional beamforming processing in frequency domain. This processings are applied here for TTW imaging. Other spectral estimation techniques have already been used for imaging [9] like in SAR, but here the implementation should take into account near field considerations and TTW propagation. Imaging with simulated numerical data for different scenarii illustrates first results.

\section{Signal Model and TTW Propagation}

In this section we will begin by giving the backscattered signal that impinges a receiving antenna array in the case of near field considerations and then show how to take into account TTW propagation. A signal model in frequency domain for a MIMO architecture is finally proposed.

\subsection{Near Field Signal Mode}

Let consider a MIMO radar system constituted of $M$ transmitting antennas located at $\left(\mathbf{x}_{\mathrm{m}}\right)_{\mathrm{m}=1 . . \mathrm{M}}=\left[\mathrm{x}_{\mathrm{m}}, \mathrm{y}_{\mathrm{m}}\right]^{\mathrm{T}}$ and $N$ receiving antennas located at $\left(\mathbf{x}_{\mathrm{n}}\right)_{\mathrm{n}=1 . . \mathrm{N}}=\left[\mathrm{x}_{\mathrm{n}}, \mathrm{y}_{\mathrm{n}}\right]^{\mathrm{T}}$. In the case of near field free space propagation spherical wave 
fronts are assumed, then the signal waveform $\mathbf{s}_{\mathrm{m}}$ emitted by the $m$-th transmitting antenna and backscattered by a target located at $\mathbf{x}_{t}=\left[\mathrm{x}_{\mathrm{t}}, \mathrm{y}_{\mathrm{t}}\right]^{\mathrm{T}}$ to receiving antennas (fig. 1) is given by the following equation:

$$
z_{m n}(f)=\frac{\alpha_{m n}(f, \mathbf{x})}{\mathrm{r}_{m t} \mathrm{r}_{t n}} s_{m}(f) e^{-j 2 \pi f \tau_{m n}(\mathbf{x})}
$$

where $f$ stands for the frequency and $\mathbf{s}_{\mathrm{m}}(f)$ the $m$-th emitted waveform frequency bin at $\mathrm{f}$. The time delay needed by the wave to go from the m-th Tx antenna to target and to return to the $n$-th $\mathrm{Rx}$ antenna is noted $\tau_{m n}(\mathbf{x})$ and $\alpha(f, \mathbf{x})$ is representative of the target radar cross section (RCS).

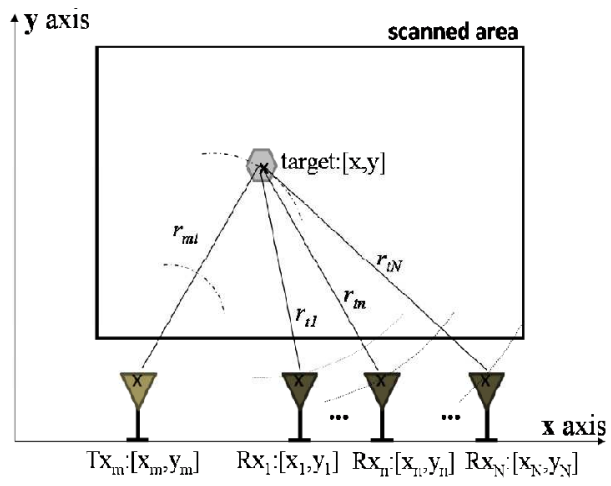

Figure 1. Spherical propagation from the m-th transmitting antenna to target and from target to the $N$ receiving antennas.

Actually the amplitude depends on both incidence (m's and n's dependency) and frequency but for simplicity in this work those dependencies will be disregarded and amplitude will be noted $\alpha(f, \mathbf{x})$. In free space, $\tau_{m n}(\mathbf{x})$ can be expressed as a function of $r_{m t}$ and $r_{t n}$, the distance between the $m$-th Tx antenna and the target respectively between the target and $n$-th $\mathrm{Rx}$ antenna and $c$ the velocity:

$$
\begin{array}{cl}
\tau_{m n}(\mathbf{x}) & =\left(\mathrm{r}_{m t}+\mathrm{r}_{t n}\right) / c \\
r_{m t} & =\left\|\mathbf{x}-\mathbf{x}_{m}\right\| \\
r_{t n} & =\left\|\mathbf{x}-\mathbf{x}_{n}\right\|
\end{array}
$$

\subsection{Through the Wall Propagation Considerations}

Through the wall wave penetrating spawn various effects [10] as reflection, transmission speed change, refraction, absorption, diffraction and wavefront distorsion, detailed explanations can be found in [10]. If processing do not take into account those effects, imaging results will degrade and resolution decreases. An ideal signal model should take into account all those effects but it is impossible in practical terms. Here we will explain how to modify the time delay to take into account refraction and speed change [11].

Let suppose that the scanned area is surrounded with walls of equivalent constant permittivity $\varepsilon_{w}$ and thickness $e_{w}$ (figure 2a). Considering for example the return path from the target to the $n$-th receiving antenna, the effective computation of the time delay $\tau_{\mathrm{n}}$ used the fact that it does not change if we set the wall external interface at the antenna position (fig. 2b). Then the travel time computation can be lead equivalently in the figure $2 \mathrm{~b}$ configuration with $h=h_{1}+h_{2}$.
The approximated value of the inflexion point $x_{b}$ is given by [11]:

$$
\mathbf{x}_{b}=\mathbf{x}_{c}+\sqrt{\frac{1}{\epsilon_{w}}}\left(\mathbf{x}_{a}-\mathbf{x}_{c}\right)=\mathbf{x}_{n}+\frac{1}{\sqrt{\epsilon_{w}}} \frac{e_{w}}{\left(h+e_{w}\right)}\left(\mathbf{x}-\mathbf{x}_{n}\right)
$$
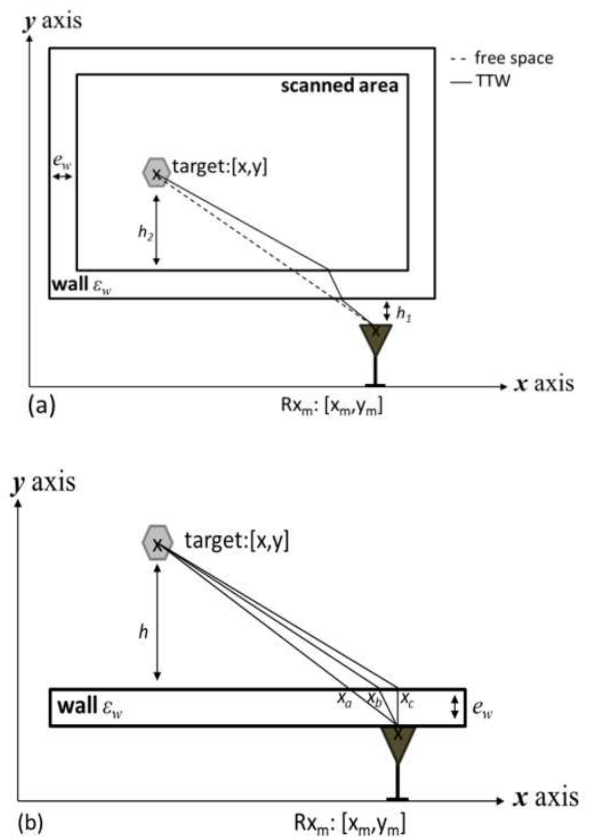

Figure 2. TTW propagation vs free space propagation.

We can now give the travel time $\tau_{n}$ in the case of TTW propagation :

$$
\tau_{n}=\frac{d_{\text {air }}}{c}+\frac{d_{\text {wall }} \sqrt{\epsilon_{w}}}{c}
$$

where $d_{\text {air }}$ represents the travel distance in air and $d_{\text {wall }}$ the travel distance in the wall. The time delay from the $m$-th transmitting antenna to the target is computed in the same way and the total time delay $\tau_{m n}(\mathbf{x})$ will be given by the sum of the two computed delays $\tau_{\mathrm{m}}(\mathbf{x})+\tau_{\mathrm{n}}(\mathbf{x})$. To introduce array notation, we rewrite:

$$
\begin{aligned}
z_{m n}(f) & =\alpha(\mathbf{x}) \frac{e^{-j 2 \pi f \tau_{n}(\mathbf{x})}}{\mathbf{r}_{t n}} \frac{e^{-j 2 \pi f \tau_{m}(\mathbf{x})}}{\mathbf{r}_{m t}} s_{m}(f) \\
& =\alpha(\mathbf{x}) \mathbf{a}_{r}(\mathbf{x}, f)_{n} \mathbf{a}_{t}(\mathbf{x}, f)_{m} s_{m}(f)
\end{aligned}
$$

Where

$$
\mathrm{a}_{t}(\mathbf{x}, f)_{m}=e^{-j 2 \pi f \tau_{m}(\mathbf{x})} / \mathrm{r}_{m t}
$$

is the steered component from the $m$-th transmitting antenna to target at $\mathrm{x}$ and

$$
\mathrm{a}_{r}(\mathbf{x}, f)_{n}=e^{-j 2 \pi f \tau_{n}(\mathbf{x})} / \mathrm{r}_{t n}
$$

this of the target to the $n$-th receiving antenna at frequency f.

\subsection{MIMO Radar Signal Model}

We consider a MIMO system with $\mathrm{M}$ transmitting and $\mathrm{N}$ 
receiving antennas. To differentiate signals coming from various transmitting antennas we can use frequency hop code waveforms as hyperbolic hop codes [12]. Thus each of the $M$ transmitted signals consists in $L$ monofrequency pulses at frequencies $\left(f_{l}\right)_{l=1 . . L}=f_{0}+l(B / L)$, where $f_{0}$ is the center frequency, $B$ the bandwidth and $L$ the number of frequency bins. Using previous introduced notations and the fact that frequency hop codes are used we can assume without loss of generality that $\boldsymbol{s}_{m}\left(f_{l}\right)_{l=1 . . L}=1$ for all transmitting antennas, the received signal at frequency fl by the MIMO array can then be written as follow:

$$
\mathbf{Z}\left(f_{l}\right)=\alpha(\mathbf{x}) \mathbf{a}_{r}\left(\mathbf{x}, f_{l}\right) \mathbf{a}_{t}^{T}\left(\mathbf{x}, f_{l}\right)+\mathbf{N}_{\text {noise }}
$$

with:

$$
\begin{aligned}
\mathbf{a}_{t}\left(\mathbf{x}, f_{l}\right) & =\left[\begin{array}{llll}
\mathbf{a}_{t}(\mathbf{x}, f)_{1} & . . & \mathbf{a}_{t}(\mathbf{x}, f)_{M}
\end{array}\right]^{T} \\
\mathbf{a}_{r}\left(\mathbf{x}, f_{l}\right) & -\left[\begin{array}{llll}
\mathbf{a}_{r}(\mathbf{x}, f)_{1} & . & \mathbf{a}_{r}(\mathbf{x}, f)_{N}
\end{array}\right]^{T}
\end{aligned}
$$

where $\mathrm{Z}\left(f_{l}\right)$ is the complex data matrix of size $N \mathrm{x} M$. The transmit array manifold at frequency $f_{l}$ is noted $\mathrm{a}_{\mathrm{t}}\left(\mathbf{x}_{f} f_{l}\right)$, the receive one $\mathrm{a}_{\mathrm{r}}\left(\mathbf{x}, f_{l}\right)$ and $\mathrm{N}_{\text {noise }}$ is additive noise. Whole data signal is obtained by concatenation of data signal matrices at different frequencies $\left(f_{l}\right)_{l=1 . . L}$ form a $3 \mathrm{D}$ complex data matrix of size $N \mathrm{x} M \mathrm{x} L$.

\subsection{Advantages of MIMO Radar for TTW Imaging}

The concept of Multiple Input Multiple Output (MIMO) comes from the field of telecommunication and has been extended to radar and is now subject to many researches in the radar community [13], [14]. A MIMO radar is a system which is constituted of an array with several transmitting and receiving antennas. Theoretical studies [15] showed that MIMO radar system could bring significant improvements of radar performances as the increase of probability of detection, cross range resolution or maximum number of detectable targets and even to synthesize transmitting beampattern adaptive to the environment. Here we present the advantages of a MIMO architecture for the task of detection and localization in the specific TTW environment. Firstly, the indoor environment present a lot of obstacles as funitures that could hide targets. This is why spatially spread transmitting antennas are required to ensure target detectability which justify our interest in a system constituted of several transmitting antennas. Then, variations of propagation channel with attenuations that could have large value degrade cross range resolution. In order to keep resolution, several points of view of the scene are needed. The spatial diversity offered by several transmitting and receiving antennas ensures differents point of view of the scene. Multipaths effects due to reflections on differents walls constituting room bring ghosts but those ghosts differs for different transmitting antennas thus overall data processing could prevent form those artefacts. And more generally, the MIMO 3D array signal matrix gives new degrees of flexibility in terms of data rearrangements and coherent or incoherent processings for robust processing implementa- tions.

\section{Radar Imaging Processing}

The objective of a radar system is to detect and localise targets in the analyzed scene. Here those objectives are realized through radar imaging processing. In the following we present a conventional beamforming processing for imaging. For notation convenience, we note $z_{l}$ the column stacked vector of $Z\left(f_{l}\right)$ usually written $\mathrm{z}_{l}=\operatorname{vec}\left[Z\left(f_{l}\right)\right]$ and noticing that $\operatorname{vec}\left[\mathrm{a}_{\mathrm{r}}\left(\mathrm{x}, f_{l}\right) \mathrm{a}_{\mathrm{t}}^{\mathrm{T}}\left(\mathrm{x}, f_{l}\right)\right]=\mathrm{a}_{\mathrm{t}}^{\mathrm{T}}\left(\mathrm{x}, f_{l}\right) \times \mathrm{a}_{\mathrm{r}}\left(\mathrm{x}, f_{l}\right)$, where $\times$ is the kronecker product, the received signal in (6) is rewritten

$$
\mathbf{z}_{l}=\alpha(\mathbf{x}) \mathbf{a}_{l}(\mathbf{x})+\mathbf{n}_{\text {noise }}
$$

the vector $\mathrm{a}_{l}(\mathrm{x})=\mathrm{a}_{\mathrm{t}}^{\mathrm{T}}\left(\mathrm{x}, f_{l}\right) \times \mathrm{a}_{\mathrm{r}}\left(\mathrm{x}, f_{l}\right)$, can be considered as a MIMO steering vector at frequency $f_{l}$.

\subsection{Conventional MIMO Beamforming Imaging}

Beamforming is a processing that tries to focus the array to signals coming from only one particular position. In practical terms for imaging processing, it means that the intensity associated at each pixel at $\mathrm{x}$ will be the output power of a beamformer filter that is steered towards the considered position. Note that in our MIMO data signal matrix the steering operation will depend not only on antennas positions but also on frequency so the processing can be done at every bin $\left(f_{1}\right)$ and sum over frequency. The output power of conventional beamforming at $f_{1}$ is expressed as:

$$
\mathrm{s}_{l}(\mathbf{x})=\left|\mathbf{w}^{H} \mathbf{z}_{l}\right|^{2}
$$

where $\mathrm{w}_{l}$ is the steered vector to position $\mathrm{x}$ at frequency $f_{l}$. In far field we would have $\mathrm{w}=\mathrm{a}_{l}(\mathrm{x})$ but in order to take into account near field we multiply by a vector $d$ that compensates the $1 /\left(\mathrm{r}_{m t} \mathrm{r}_{t n}\right)$ attenuation and $\mathrm{w}$ is given by the element wise multiplication of $d$ by $a_{l}(x)$. Then the intensity given at each pixel at location $\mathrm{x}$ is simply $\mathrm{s}(\mathrm{x})=\sum \mathrm{s}_{l}(\mathrm{x})$. Time averaging is often used to reduce noise effects and image artefacts

$$
\mathrm{s}_{l}(\mathbf{x})=\left\langle\left|\mathbf{w}^{H} \mathbf{z}_{l}\right|^{2}\right\rangle
$$

where $<.>$ denotes the expected value or time average.

\section{Simulations and Results}

For numerical examples we use a MIMO radar architecture with $M=5$ transmitting antennas and $N=15$ receiving antennas. We consider a MIMO configuration where the receive array is a 0.5 -wavelength spaced uniform linear array (ULA) and the transmit array is a 1.5-wavelength spaced ULA. The frequency bandwidth is $B=800 \mathrm{MHz}$, the center frequency $f_{0}=1 \mathrm{GHz}$ and number of frequency bin $L=88$. The antennas are modeled by dipole antennas calibrated at the center frequency and the wall modelling has 
been made with $20 \mathrm{~cm}$ depth $40 \mathrm{~cm}$ width cinder blocks fig. (3a) of permittivity $\varepsilon_{w}=4$, view of the room is given in fig. (3b). The whole scene consists in a $4.7 \mathrm{~m}$ width $4.25 \mathrm{~m}$ depth room. The scenario considered were an empty room with one or two cylindrical targets fig. (4a). To efficiently take into account TTW propagations effects scene modelling has been made as realistic as possible and data signals were obtained through full wave FDTD computation. Modelling and FDTD computations have been made with the commercial software CST MicroWave Studio.

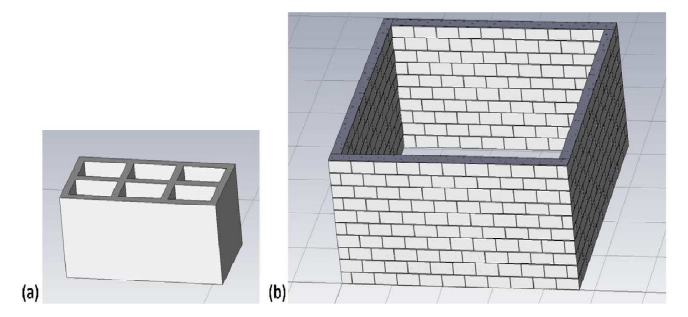

Figure 3. Wall modeling with cinder block in (a) and view of the modeled room in (b).

Direct application of conventional beamforming does not allow targets detection because strong reflections of walls conceal the backscattered signals from target inside the room. Thus subtraction by target free signals is first done before processing. The images obtained for one and two targets by the MIMO system are presented in fig. (4). We can see that the proposed method allows targets detection at the correct locations because of the fact that we take into account speed change end path delays due to TTW propagation. But left and right ghosts caused by first reflexions on wall interfaces still persist nevertheless there are about $15 \mathrm{~dB}$ below target's mainlobe then thresholding could facilitate detection and localization. Target spot spreading is first due to resolution limitation but not only. In fact as some of TTW propagation effects are not considered in our signal model, as multiple reflections and interferences inside the wall, thus imaging performances are degraded. Further analysis in term of point spread function deterioration due to TTW propagation should be led.

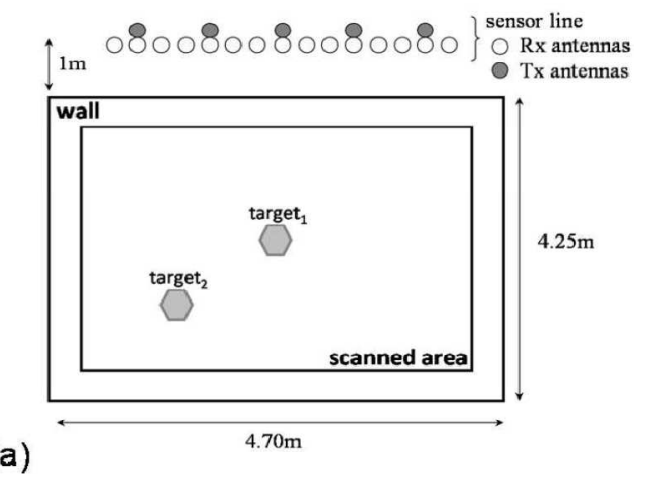

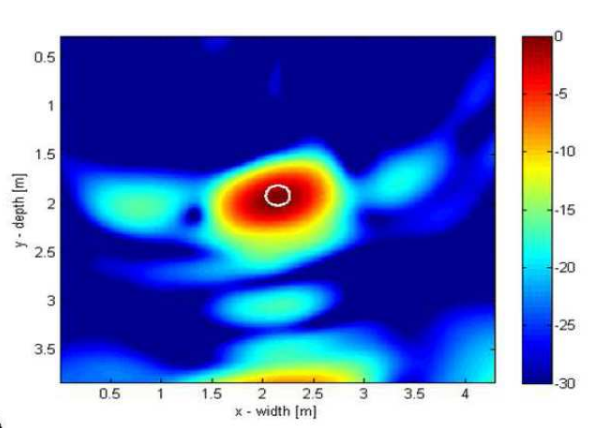

(b)

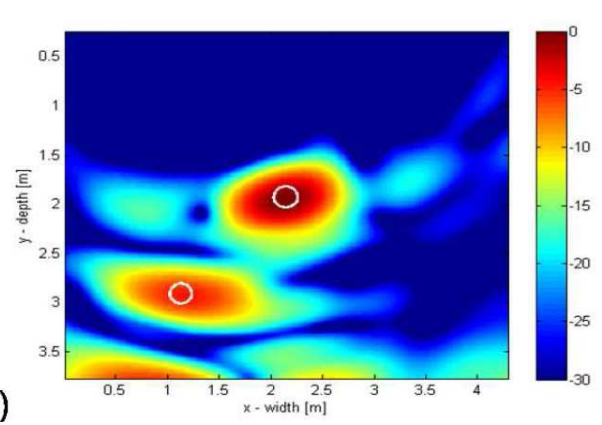

Figure 4. TTW imaging results on synthetic data with MIMO conventionnal beamforming for one and two targets scenarii.

\section{MIMO Radar Prototype and Expe- rimental Résults}

To complete the previous study [1], an experimental setup of MIMO radar for TTW detection has been realised in our laboratory. To allow differentiation between signals coming from the transmitters, time multiplexing have been used. Thus electronic switches have been used to address the different transmitters and receivers. The receiver has an homodyne receiver architecture for its better clutter rejection capability and higher sensitivity compared to heterodyne scheme. So, on receive, a coupler extracts a part of the transmitted and mix it to the received signal. Some other components for amplification and filtering are also added. The source is an yttrium iron garnet (YIG) oscillator permitting fast frequency sweeping through a large bandwidth. The YIG and switch controls are voltage commands realised by an FPGA for high time precision. The synoptic of the radar system is given fig. 5

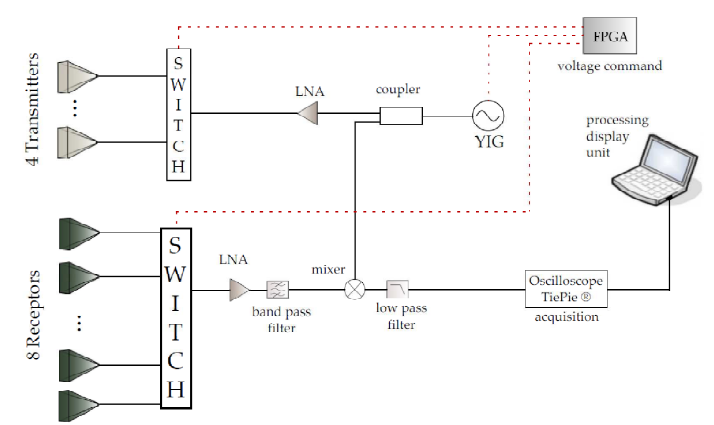

Figure 5. Overview of time multiplexed ultrawide band MIMO radar. 
The antennas are ultrawide band ETSA antennas that have been realised by the Electronics Antennas and Telecommunications Laboratory (LEAT) of the University of Nice Sophia Antipolis. Transmission and reception is in vertical polarization. The waveform is a linear frequency modulation (LFM) from $2 \mathrm{GHz}$ to $4 \mathrm{GHz}$. A picture of the radar system is given fig. 6 .

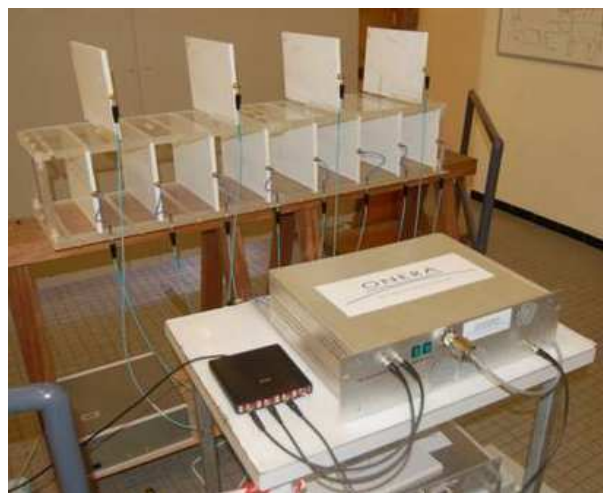

Figure 6. Picture of the realised time multiplexed ultrawide band MIMO radar.

The realised radar has $M=4$ transmitting antennas and $N=8$ receiving antenna. The total time scan of the scene is a snapshot of $T=8 \times 4 \times 2 \mathrm{~ms}=64 \mathrm{~ms}$. The data acquisition is realised with a digital oscilloscope Tiepie which is connected to a computer through USB. The constructor also provides dynamic link librairy (dll) to directly retrieve data. The summary of the radar characteristics are given in the table below.

\begin{tabular}{|ll|}
\hline \multicolumn{2}{|c|}{ MIMO radar characteristics } \\
\hline number of transmitters & 4 \\
number of receivers & 8 \\
polarization & vertical on transmit and receive \\
waveform & LFMCW from 2 to $4 \mathrm{GHz}$ \\
frequency band & $2 \mathrm{GHz}$ \\
emitted power & $17 \mathrm{dBm}$ \\
multiplexing & time multiplexing \\
scan time & $64 \mathrm{~ms}$ \\
& \\
\hline
\end{tabular}

Radar measurements have been carried out through a cinder brick wall as illustrated fig.8. The antenna array is placed at $1 \mathrm{~m}$ in front the wall. The target consists in a metallic plate of $30 \mathrm{~cm}$ height $20 \mathrm{~cm}$ witdh placed behind the wall at differents positions. Some results of imaging are given in fig.9. The right target position is marked by a white circle in the obtained image. Compared to simulation results, range resolution of measurements are higher as experimental bandwitdh is $2 \mathrm{GHz}$ which is more than twice the bandwidth used in simulation. This difference is related to the fact that [1] was published earlier and before the radar system characteristics settled. We can see that through cinder block wall imaging suffered from strong artefacts. This is due to the fact that heterogeneous wall causes partial wavefront destruction resulting in some high intensity around target position. We can also notice bias on target position due to difference in wall permittivity. In fact cinder block wall is an heterogeneous wall with holes inside and making the approximation of a homogeneous wall with a global permittivity brings some errors. Despite these drawbacks, target can approximatively be localised at the right positions and adequate thresholding going along with tracking processing could mitigate TTW imaging artefacts. Next investigations are lead on other radar imaging algorithms and tracking.
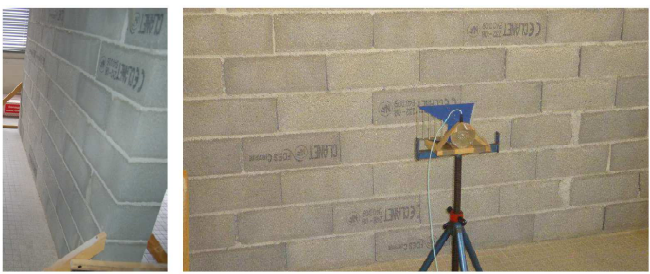

Figure 7. Cinder block wall used for experimentation.
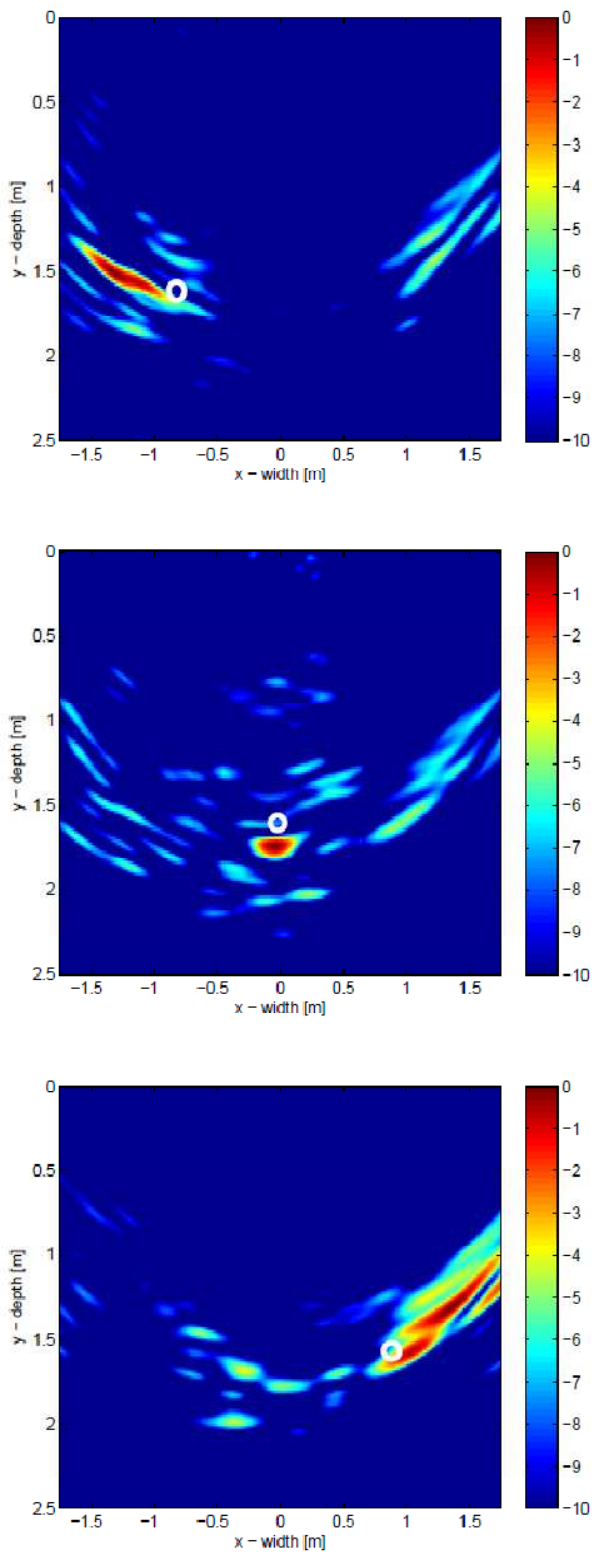

Figure 8. Experimental results of imaging through a cinder block wall with the MIMO radar system. 


\section{Conclusion}

In this work, we have presented a frequency backscattered signal model that takes into account some of the TTW propagation effects on electromagnetic wave as refraction and speed change and also near field considerations. The main problems encountered in TTW radar detection are addressed and advantages of a MIMO architecture for TTW imaging is explained. After what a MIMO frequency signal model is proposed. Then imaging processing is derived with a conventional beamformer approach. To illustrate the feasability of detection, imaging processings were implemented with FDTD simulations data for different scenarii with one or two targets inside in the case of cinder block walls. We have shown that taking into account propagation delay allow to localize the target at the correct location but as some of effects of TTW propagation effects can not be mitigated image performance are degraded. Nevertheless conventional beamforming is robust enough to allow detection and localization. Experimental results have also been presented. First the realised MIMO radar have been described then imaging results through a cinder block wall have been given. Despite small imaging artifacts, thresholding and tracking processing could be a solution for efficient target localization. Future works for antenna arrangement and comparison to existing radar architecture as SAR are on investigation on top of other processing for better resolution.

\section{Acknowledgements}

The main part of this study is funded by a $\mathrm{PhD}$ from the DGA. The authors would like to thank DGA for its support.

\section{References}

[1] B. Boudamouz, P. Millot, C. Pichot, C., "Through the wall radar imaging with MIMO beamforming processing". Microwaves, Radar and Remote Sensing Symposium (MRRS). pp.251-254, 25-27 Aug. 2011.

[2] M. Farwell, J. Ross, R. Luttrell, D. Cohen, W. Chin and T. Dogaru. "Sense through the wall system development and design considerations". Journal of the Franklin Institute, Number 6 Vol. 345, p. 570-591, 2008.

[3] Edward J. Baranoski. "Through-wall imaging: Historical perspective and future directions". Journal of the Franklin
Institute, Number 6 Vol. 345, pp. 556-569, 2008.

[4] L. M. Frazier. "Surveillance through walls and other opaque materials". Aerospace and Electronic Systems Magazine, IEEE , vol.11, no.10, pp.6- 9, Oct 1996.

[5] A. R. Hunt, "A wideband imaging radar for through-the-wall surveillance". Proceedings SPIE Sensors, and Command, Control, Communications, and Intelligence (C3I) Technologies for Homeland Security and Homeland Defense III, vol. 5403, p. 590-596, 2004.

[6] F. Ahmad and M. G. Amin. "Noncoherent Approach to Through-the-Wall Radar Localization". IEEE Transactions On Aerospace And Electronic Systems, vol. 42, p. 1405-1419, 2006.

[7] L. Li, W. Zhang, F. Li. "A Novel Autofocusing Approach for Real-Time Through-Wall Imaging Under Unknown Wall Characteristics". Geoscience and Remote Sensing, IEEE Transactions on , vol.48, no.1, pp.423-431, Jan. 2010.

[8] Y.S. Yoon and M. G. Amin. "High resolution through-the-wall radar image based on beamspace eigenstructure subspace methods". Proc. SPIE 6947, 69470C (2008).

[9] S.R. DeGraaf, "SAR imaging via modern 2-D spectral estimation methods". Image Processing, IEEE Transactions on, vol.7, no.5, pp.729-761, May 1998.

[10] L.M. Frazier, "MDR for law enforcement [motion detector radar] Potentials". IEEE, vol.16, no.5, pp.23-26, Dec 1997/Jan 1998

[11] H. Dong-Mei and Z. Qin-Yu, "Impulse radio ultra-wide-band through wall imaging radar based on multiple-input multiple-output antenna arrays". Information Technology Journal, vol. 9, pp. 782789, 2010.

[12] S. Maric and E. Titlebaum, "A class of frequency hop codes with nearly ideal characteristics for use in multiple-access spread-spectrum communications and radar and sonar systems". IEEE Trans. Commun., vol. 11, pp. 14421447, Sept. 1992.

[13] E. Fishler, A. Haimovich, R. Blum, L. Cimini, D. Chizhik, and R. Valenzuela, "Mimo radar: an idea whose time has come". Radar Conference Proc. of the IEEE, pp. 7178, 2004.

[14] F. C. Robey, S. Coutts, D. Weikle, J. C. McHarg, and K. Cuomo, "Mimo radar theory and experimental results". Signals, Systems and Computers, Conf. Rec. of the 38th Asilomar Conf. on, pp. 300304, 2004.

[15] J. Li and P. Stoica MIMO Radar Signal Processing. Hardcover: Wiley,2008. 